\title{
Analysis on Skin Colour Model Using Adaptive Threshold Values for Hand Segmentation
}

\author{
Phyu Myo Thwe ${ }^{1}$ \\ ${ }^{1}$ Image Processing Lab, University of Computer Studies, Mandalay, Myanmar \\ Email: phyumyothwe@ucsm.edu.mm \\ May The' $\mathbf{Y u}^{2}$ \\ ${ }^{2}$ Faculty of Information Science, University of Computer Studies, Mandalay, Myanmar \\ Email: maytheyu@ucsm.edu.mm
}

Received: 26 June 2019; Accepted: 07 August 2019; Published: 08 September 2019

\begin{abstract}
The hand gesture recognition system is the hottest topic for the human-machine interaction and computer vision fields. The hand gesture recognition system is still a challenging research area in computer vision for human-computer interaction because of various device conditions, various illumination effects, and very complex background. The recognition of hand gestures used in various application areas: such as sign language recognition, man-machine interaction, human-robot interaction, and intelligent device control and many other application areas. The robust detection of hand in hand gesture recognition system has become a challenging task due to clutter background, dynamic background, and various illumination conditions in real-world conditions. Segmentation is the partioning/separating the foreground hand region from the background region in an image. Segmentation is also pre-processing steps of the hand gesture recognition system. The recognition accuracy will increase if the hand region correctly detected. So, hand region detection is the main important step for the hand gesture recognition system.
\end{abstract}

Index Terms-Hand Detection, Hand Gesture Recognition, Colour Segmentation, Human Computer Interaction.

\section{INTRODUCTION}

Hand gesture segmentation is the hottest topic in manmachine interaction and computer vision. Segmentation is the process of separating the background and foreground. The objective of hand segmentation is to find out the hand region from the images region or no-hand region. There are many hands segmentation techniques such as threshold-based, edge-based, region-based, pixel-based, model-based, ANN-based, watershed-based, and clustering-based and many other segmentation methods. In all of these techniques, the skin colour threshold based method is simple and also common used method in stateof-art research. But, skin colour thresholds based method remains challenging when background contains the other objects like the human skin colour. Firstly, in this paper,
YCbCr colour threshol ds based approach is implemented. $\mathrm{YCbCr}$ colour space has good characteristics such as colour independent each colour components, good colour clustering and simple to calculate. The main advantages of skin colour based segmentation are (i) it is easy and fast to implement in training and testing. (ii) It is not dependent on the distribution of neighbour colour pixels. The strength of hand segmentation for hand gesture recognition can be reducing the amount of data or the dimension of data values. In this paper, the authors are experimented with various threshold values on $\mathrm{YCbCr}$ colour space to detect the human skin.

The main challenges that need to solve as research issue include:

- Illumination condition is the most sensitivity for hand gesture recognition of vision based system.

- Complex backgrounds, dynamic backgrounds are also main difficult for hand gesture recognition.

- The different size of the user's hand is another issue.

- The multiple gestures in the same background and different viewpoints.

- The several persons contains in the sense other than the real subjects.

- The occlusion problem can also occur for the hand gesture recognition problem.

- Devices are also important to capture the images because different cameras have the different output for the same images.

- In dynamic gestures, the movement of hand can degrade the quality of images due to the blurring conditions.

The motivation of our research is to find out the best threshold values of $\mathrm{YCbCr}$ colour space for human skin detection. $\mathrm{YCbCr}$ color space is the best colour model than HSV and RGB in colour clustering range and colour independent for skin segmentation [9,17,18,21]. According to the previous literature, $\mathrm{YCbCr}$ colour space is the best suitable colour space for human skin detection. But, the colour spaces and threshold values are not fixing 
because different taking image devices, skin tone, illumination changing, blurring condition due to the movement object, shadow and many other conditions.

The general steps of hand gesture recognition system describe the following Fig 1. In Pre-Processing step performs image resizing, image debluring, image enhancement and so on. In hand region detection, extract the hand from the background region by using human skin colour, background subtraction method, foreground extraction and many other methods. In feature extraction step, extract the hand gesture features by using various deep learning pre-train models; supervise learning and unsupervised learning methods. The most common features are colour features, edge features, shape features, orientation features, location features and many other specific features.

The rest of the paper is described as following: Section II describes the detail explanation of the literature survey and Section III presents the ways of data collection for hand gestures. Section IV describes about the datasets. Section V explains the YCbCr colour model. The experimental results are described in section VI. The last section describes the conclusion and future work of the research.

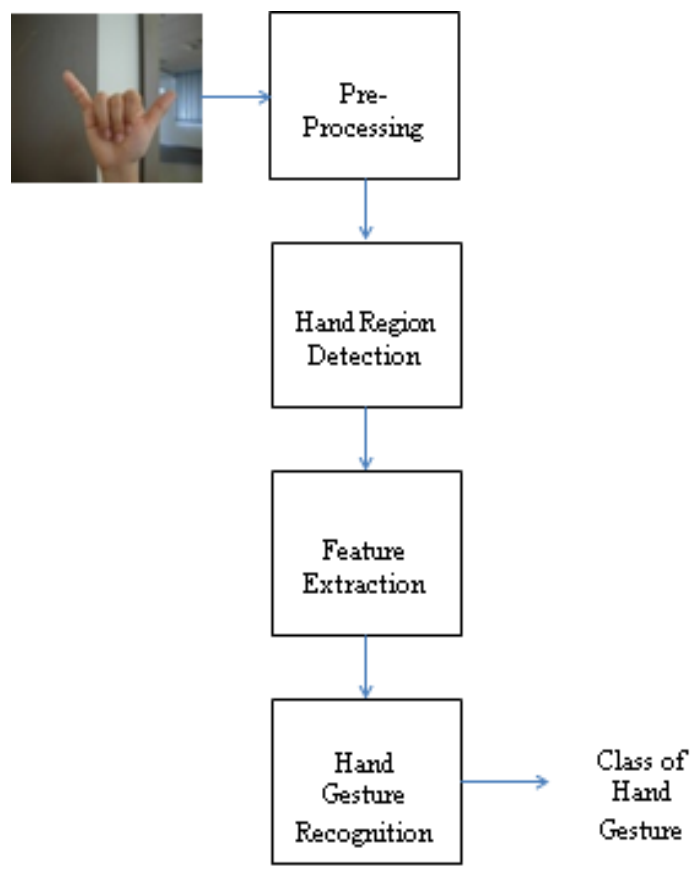

Fig.1. General Hand Gesture Recognition Steps

\section{RELATED WROKS}

Although many researchers have solved the issues of hand segmentation from the very clutter background, the robust algorithm is still needed to reconcile in any conditions such as various illumination conditions, dynamic background, static clutter background, etc. Shaik et al [1] implemented $\mathrm{YCbCr}$ colour thresholds based approach. In this paper, the authors applied the threshold values between $150 \& 200$ for chrominance component
$\mathrm{Cr}$ and the chrominance components $\mathrm{Cb}$ are between 100 $\& 200$. But, the threshold value is not suitable to detect the hand in NUS hand pose dataset I. McBride et al (2019) proposed skin segmentation/ detection algorithms to recognize the hand gesture of the hand gesture recognition system. The authors used $\mathrm{YCbCr}$ skin thresholding based algorithm, RGB-H-CrCb skin thresholding based algorithm and KNN skin classification algorithm. In this proposed study, the author used chrominance component $\mathrm{Cb}$ and $\mathrm{Cr}$ values between 80 \& 120 and $133 \& 173$. The authors tested the segmented results on the three different particular images and compare these three different algorithms. But, these threshold values for $\mathrm{CbCr}$ are not suitable to segment the hand in NUS Hand Pose datasets I [2].

Nguyen et al (2018) introduce the new hand segmentation by combining the Mask R-CNN and the tracking. The original Mask R-CNN can't detect the hand when the blur condition due to the motion. The author's proposed method produced a better result than the original Mask R-CNN [3].

Zhang et al (2018) implemented novel hand segmentation by combining saliency detection and skin colour detection. The implemented method is more robust than state-of-art research in complex background [4]. Rahmat and his partners (2016) proposed skin colour segmentation by combining three different colour spaces such as Normalized RGB, HSV and YCbCr colour space. The skin colour detection accuracy is more robust than the single colour spaces. The recognition accuracy was obtained $91.05 \%$ on two different datasets (ECU and HGR) [5].

Thakur and his friends (2011) study that novel skin colour detection model by combining RGB, HS and $\mathrm{CbCr}$ and named as RGB-HS-CbCr to detect the human face. The proposed model can successfully detect the human skin regions and human non-skin regions [6]. Yingxin et al (2016) presents a robust hand gesture recognition method via convolutional neural network. In this paper, the authors firstly pre-process on the hand gesture input by using canny edge detection and then extracted features by using CNN. The accuracy of the experimental results on original the input image is lower than the accuracy of the edge extracted images. Canny edge detection can be used to eliminate the illumination variation on the original input image [15].

Phaung et al (2003) implemented skin segmentation algorithm by combining colour and edge information. In this paper, the authors tested five different colour spaces such as YCbCr, HSV, RGB, CIE XYZ and CIE Lab [8]. Phaung et al (2005) also have written the next paper for human skin colour segmentation on four different colour spaces such as CIE-Lab, YCbCr, HSV and RGB colour spaces. [7].

Rungruangbaiyok et al (2015) proposed skin colour threshold-based segmentation methods with the optimized threshold values on various colour models. In this paper, the ensemble Threshold Segmentation method (ETS) with the HSV colour space model is the most accurate method than other colour space threshold based 
methods. The threshold values of all colour spaces are between 0 and 1 . The accuracy was obtained $98.51 \%$ on NUS Hand Pose Dataset I. [14].

Wang et al (2012) implemented hand segmentation methods by combining skin colour and background information. The performance of the proposed system is better than the single skin colour model [12]. Xu et al (2017) proposed the hand region identification algorithm by using $\mathrm{YCgCr}$ colour space model. $\mathrm{YCgCr}$ colour space is based on $\mathrm{YCbCr}$ colour space and also similar [11]. Patidar et al [2013] proposed that hand segmentation and tracking by using mix colour model such as (YCbCr+HSV) and (YCbCr+RGB) [13].

Tabassum et al (2010) implemented statistical skin detection algorithms for sub-Continental Human Images. In this paper, the authors implemented with three different colour models (HSV, YUV, YUV and YIQ). According to the experimental result, the HSV colour space model is the best performance models than other two colour space models to Indian sub-continental skin colour recognition [16]. Deng et al (2011) proposed real time hand gesture recognition by using cost matrix and context based matching approach. In this paper, the authors used YCbCr skin colour model to detecting the human hand. In the experimental results, the precision rates are between $70 \%$ and $90 \%$ [17].

Ahlvers et al (2005) implemented face detection and head tracking with morphological hole mapping without using model. In this paper, the authors used three different colour modes (RGB skin cluster, YCbCr skin cluster and HSV skin cluster model) [18]. Lee et al. (2018) implemented static hand gesture recognition using wristband-based contour features. Wristband detection and skin color detection used to detect the hand and watershed segmentation and also used region merging techniques to remove the overlap condition. Finally, Minimum cosine distance used to recognize the static hand gestures. In this paper, the classification accuracy is up to $99.31 \%$ for 29 gestures. The general issue of this paper is difficult to detect the wristband when the background contains dark color and dark shadows within the gesturing hand.[19]

Sahoo et al. (2018) used grey world algorithm to remove illumination variation from the input hand gesture images and discrete wavelet transform and fisher ration (F-ration) used to extract features. Finally, the hand gestures recognized based on Support Vector Machine Algorithm. This paper is experimented on three different datasets such as MUDB dataset, Jochen-Triesch dataset and Self-Constructed dataset. The mean accuracy was obtained $98.64 \%, 95.42 \%$ and $99.08 \%$ respectively. The issues of this paper can be misclassifying gestures vocabulary when rotation noise above 15 degree. [20].

\section{Data Collation For Hand GeStures}

There are basically three ways to collect the raw data for input of hand gesture recognition system.

- The first one is applied colour glove or data glove to collect the raw hand data called glove based approach. In data glove approach, the main drawbacks are expensive because sensor node, heavily, naturalness by using data glove. The weakness of glove user has to wear the glove every time. But, the colour gloves are inexpensive; no sensors are embedded in or outside the gloves and robust method for hand gesture recognition system.

- The second way used one or more camera to collect raw input hand gesture called vision based approach. The strength of this approach is natural and more convenient for communication. But, easily affected by complex background.

- The last approach is hybrid approach to collect raw hand data by combining the above two methods.

\section{DATASETS}

NUS Hand Pose Dataset I consists of 10 classes of posture, 24 samples per class with uniform simplest background condition.

NUS Hand Post Dataset II [4] is a dataset for hand gesture recognition with complex background condition. NUS hand pose dataset II are defined 10 different static gestures of 750 hand images with human noise, the hand with 2000 hand images, and 2000 background images. In this dataset, each gesture has 5 times with 40 different subjects. The age ranges of 40 subjects are 22 to 56 years. The class types of gestures are alphabet character a to $\mathrm{j}$. The posture images take form 40 subjects and the size of images $(160 * 120)$. All the images are RGB colour space and the format of the images are .jpeg.

The sample frames of these two datasets as shows in Fig. 2.
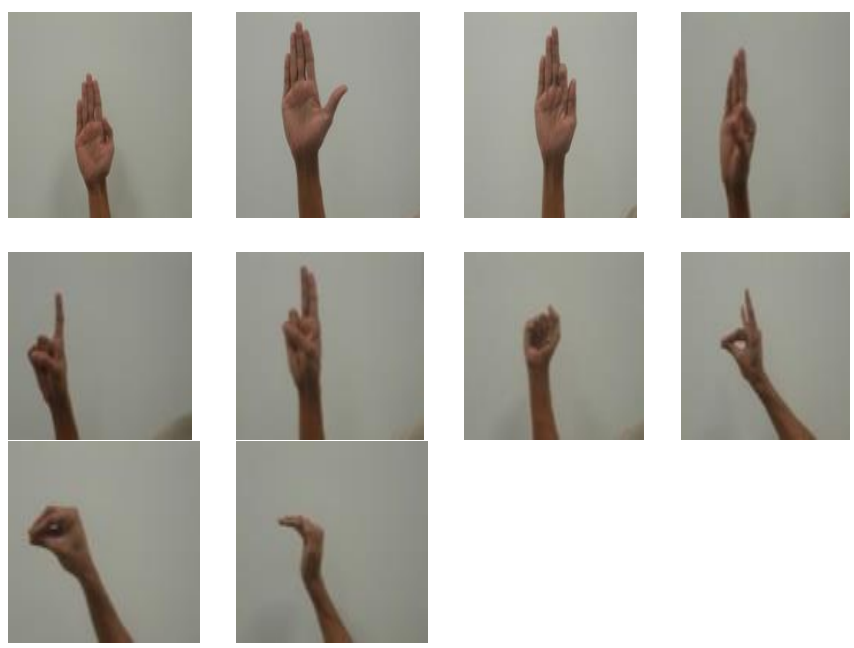

(a) NUS Hand Post Dataset I 

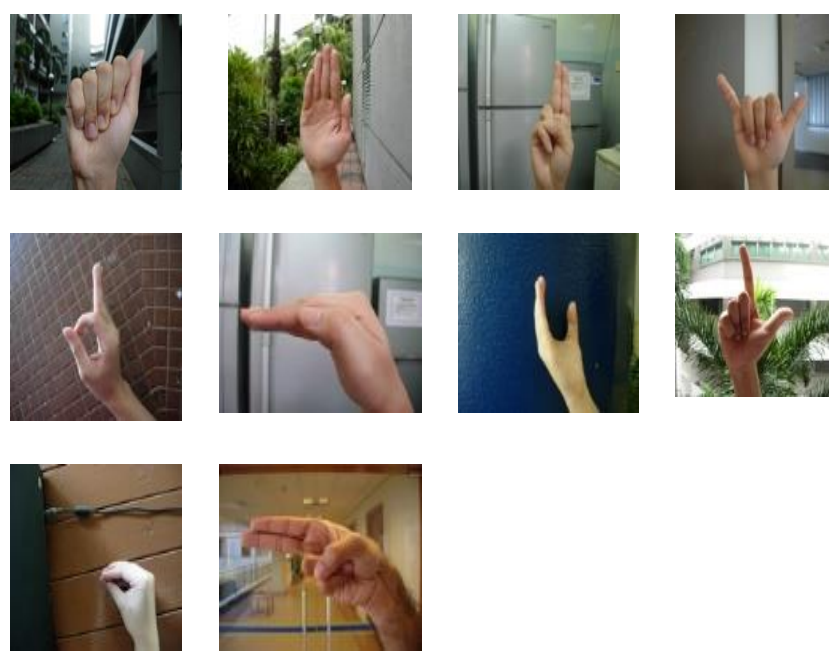

(b) NUS Hand Post Dataset II

Fig.2. Some Sample Hand Gesture Images form two different datasets

\section{YCBCR COLOUR SPACE}

There are many colour spaces in digital image processing such as RGB, HSV, YCbCr, Lab, YIQ, CMYK and many others. We should note the definition about these colour space models which are mostly used in image processing field:

According to the state of art research, $\mathrm{YCbCr}$ is the most suitable for human skin colour detection. $\mathrm{YCbCr}$ is more accurate than RGB in clustering characteristic. In $\mathrm{YCbCr}$ colour space, the channel $\mathrm{Y}$ can eliminate to improve the performance in various illumination conditions and also reduce the dimension than RGB. HSI and other colour space also eliminate the luminance component but $\mathrm{YCbCr}$ colour space has better advantages. $\mathrm{Y}$ describes the colour luminance or colour brightness and more sensitive for the human eyes. $\mathrm{Cb}$ and $\mathrm{Cr}$ is the chrominance component of the colour and less sensitive for the human eyes. The calculation process of $\mathrm{YCbCr}$ is very simple. $\mathrm{YCbCr}$ skin colour based segmentation is explained in $[1,2,5,9,10]$.The $\mathrm{YCbCr}$ colour model is shown in Fig 2. In our experiment, firstly convert RGB to $\mathrm{YCbCr}$ by using the following equation (1).

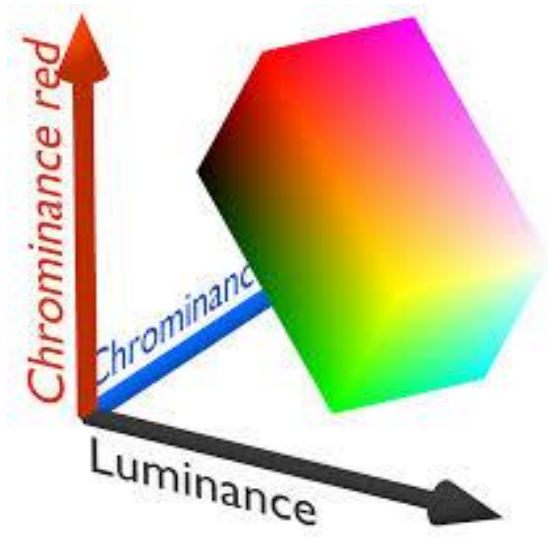

Fig.3. The YCbCr Colour Space Model

$$
\left[\begin{array}{c}
Y \\
C b \\
C r
\end{array}\right]=\left[\begin{array}{c}
16 \\
128 \\
128
\end{array}\right]+\frac{1}{256}\left[\begin{array}{ccc}
65.738 & 129.057 & 25.064 \\
-37.945 & -74.494 & 112.439 \\
112.439 & -94.154 & -18.285
\end{array}\right]\left[\begin{array}{c}
R \\
G \\
B
\end{array}\right]
$$

\section{EXPERIMENTAL SECTION}

$\mathrm{YCbCr}$ colour space is depending on the threshold values. In our experiment, we applied the threshold values (i) chrominance components $\mathrm{Cr}>133 \& \& \mathrm{Cr}<173$ and $\mathrm{Cb}>77 \quad \& \& \quad \mathrm{Cb}<127 \quad[10]$, (ii) chrominance components $\mathrm{Cr}>133 \& \& \mathrm{Cr}<173$ and $\mathrm{Cb}>80$ and $\mathrm{Cb}<120$ [2], (iii) luminance component $\mathrm{Y}>80 \& \&$ chrominance component $\mathrm{Cr}>135 \quad \& \& \quad \mathrm{Cr}<180$ and chrominance component $\mathrm{Cb}>85 \& \& \mathrm{Cb}<135$ [9]. The values ranges of $\mathrm{YCbCr}$ are 0 to 255. The different threshold values of YCbCr had been tested on NUS Hand Pose Dataset I with uniform background. In Fig3, shows original RGB images, $\mathrm{YCbCr}$ converted images, segmented black and white images with different threshold values of each hand gestures symbols with simplest background. The black and white images transformed based on user-defined threshold values by using equation 2 . According to the experimental result, all the gesture symbols in NUS Hand Pose Dataset I can correctly segment the hand region.

The first column is Original image and, the second column is $\mathrm{YCbCr}$ Conversion Image and the next columns are hand Segmentation Image with different thresholds by using $\mathrm{YCbCr}$ colour space. The same threshold values of $\mathrm{YCbCr}$ are also tested on NUS Hand Pose Dataset II with a complex background. The result shows in Fig.4. In the following Fig 4 show tested results with different threshold values on each symbols a to $\mathrm{j}$ with complex background conditions. This figure results show some of the complex background image are not suitable, mis-detected the hand from the background imges. According to the experimental results, the colour based threshold values $(77<\mathrm{Cb}<127 \& \& 133<\mathrm{Cr}<173)$ of $\mathrm{YCbCr}$ are more suitable for a simple background. But, the result may be miss detection the human hand when the backgrounds contain objects like human skin colour. In complex background condition, only $\mathrm{YCbCr}$ colour space can't correctly separate the human hand from the background.

In the previous literature, the researchers used in different colour spaces and various threshold values for human skin colour detection. Skin colour thresholding based method is very popular, easy to implement, and most often used to detect/segment the human skin. The threshold value for skin colour is difficult to set uniformly because of different types of skin colour tone, various illumination conditions. According to the start-ofart research, the different threshold values of $\mathrm{YCbCr}$, RGB, Normalized RGB, HSV colour spaces have been used for human skin segmentation. In this paper, only YCbCr colour space has been used to segment the human hand as shown in the following Table 1. 


$$
I(b, w)=\left\{\begin{array}{c}
1 ; C b(77,127) \& \& C r(133,173) \\
0 ; \text { otherwise }
\end{array}\right.
$$

Where $\mathrm{Y}, \mathrm{Cb}, \mathrm{Cr}=[0 ; 255]$ and $0=$ black and $1=$ white;

Table 1. Different Threshold Values for Human Skin Colour Detection

\begin{tabular}{|c|c|c|c|c|}
\hline References & Thresholds Values of Colour Channel & Application Domain & Colour Space & Datasets \\
\hline Shaik et al (2015) [1] & $(100<\mathrm{Cb}<150) \& \&(150<\mathrm{Cr}<200)$ & $\begin{array}{l}\text { Human Skin Colour } \\
\text { Segmentation }\end{array}$ & $\mathrm{CbCr}$ & $\begin{array}{c}\mathrm{I} \\
\text { nternet Source }\end{array}$ \\
\hline McBride et al (2019) [2] & $(80<\mathrm{Cb}<120) \& \&(133<\mathrm{Cr}<173)$ & $\begin{array}{l}\text { Human Skin Colour } \\
\text { Segmentation }\end{array}$ & $\mathrm{CbCr}$ & $\begin{array}{c}\text { Pratheepan } \\
\text { Dataset }\end{array}$ \\
\hline Rahmat et al (2004) [5] & $\begin{array}{l}\text { Normalized R/Normalized } \mathrm{G}>1.185 \& \\
0.2<\mathrm{S}<0.6 \& 0<\mathrm{H}<25 \text { or } 335<\mathrm{H}<360 \\
\quad(77<\mathrm{Cb}<127) \& \&(133<\mathrm{Cr}<173)\end{array}$ & $\begin{array}{l}\text { Human Skin Colour } \\
\text { Segmentation }\end{array}$ & $\begin{array}{l}\text { Combining } \\
\text { Normalized RGB, } \\
\text { HSV, CbCr }\end{array}$ & $\begin{array}{l}\text { ECU, HGR } \\
\text { Datasets }\end{array}$ \\
\hline $\begin{array}{l}\text { KuKharev et al (2004) } \\
{[9]}\end{array}$ & $(\mathrm{Y}>80) \& \&(85<\mathrm{Cb}<135) \& \&(135<\mathrm{Cr}<180)$ & Face Recognition & $\mathrm{YCbCr}$ & - \\
\hline Chai et al (1999) [10] & $(77<\mathrm{Cb}<127) \& \&(133<\mathrm{Cr}<173)$ & Face Segmentation & $\mathrm{CbCr}$ & - \\
\hline Deng et al (2011)[17] & $\begin{array}{c}(45<Y<180) \\
\& \&(126<\mathrm{Cb}<143) \& \&(122<\mathrm{Cr}<130)\end{array}$ & Hand Segmentation & $\mathrm{YCbCr}$ & $\begin{array}{l}\text { images from } \\
\text { CCD Camera }\end{array}$ \\
\hline Ahlvers et al (2005)[18] & $\mathrm{Y}>80 \& \&(85<\mathrm{Cb}<135) \& \&(135<\mathrm{Cr}<180)$ & Face Segmentation & $\mathrm{YCbCr}$ & $\begin{array}{l}\text { Real Time Web } \\
\text { Cam Images }\end{array}$ \\
\hline
\end{tabular}

\section{CONCLUSION AND FUTURE WORK}

Hand Gesture Recognition is one of the requirement as the language between man and machine.Hand Segmentation is the most important step of hand gesture recogniton system. There are many segmentation methods. Among them, colour based segmentation is fast processing and aslo good feature to detect the human hand. If the accurately segment the hand from the background, then can correctly recognitize the gesture. In this study, analyze $\mathrm{YCbCr}$ colur space hand segmentation algorithm on two different NUS hand pose dataset I with the simplest background and NUS hand pose dataset II with complex background and human noise background. Colour is the most important reason that lead to recognize and discriminate information. The threshold based skin colour detection algorithms mainly impact on illumination, various taking image devices, objects like human skin colour and ethnicity. The current issue on $\mathrm{YCbCr}$ threshold-based segmentation cannot correctly recognize the hand when the background contains like human skin. In the future, we will consider mix-colour space model instead of using only $\mathrm{YCbCr}$ colour space model and then we will study attention on hand segmentation with deep learning.

\section{DECLARATION}

All author(s) have disclosed no conflicts of interest. The project was self-funded.

\section{ACKNOWLEDGMENT}

Firstly, we would like to thanks our supervisor Dr. May The' Yu. She gives me valuable suggestion and mind strength for this research. The authors would like to thank Dr.Shinha for the carefully guide lining to write research paper and also the anonymous reviewers and the editor for their valuable comments and suggestions, which improvement the quality of this paper. 

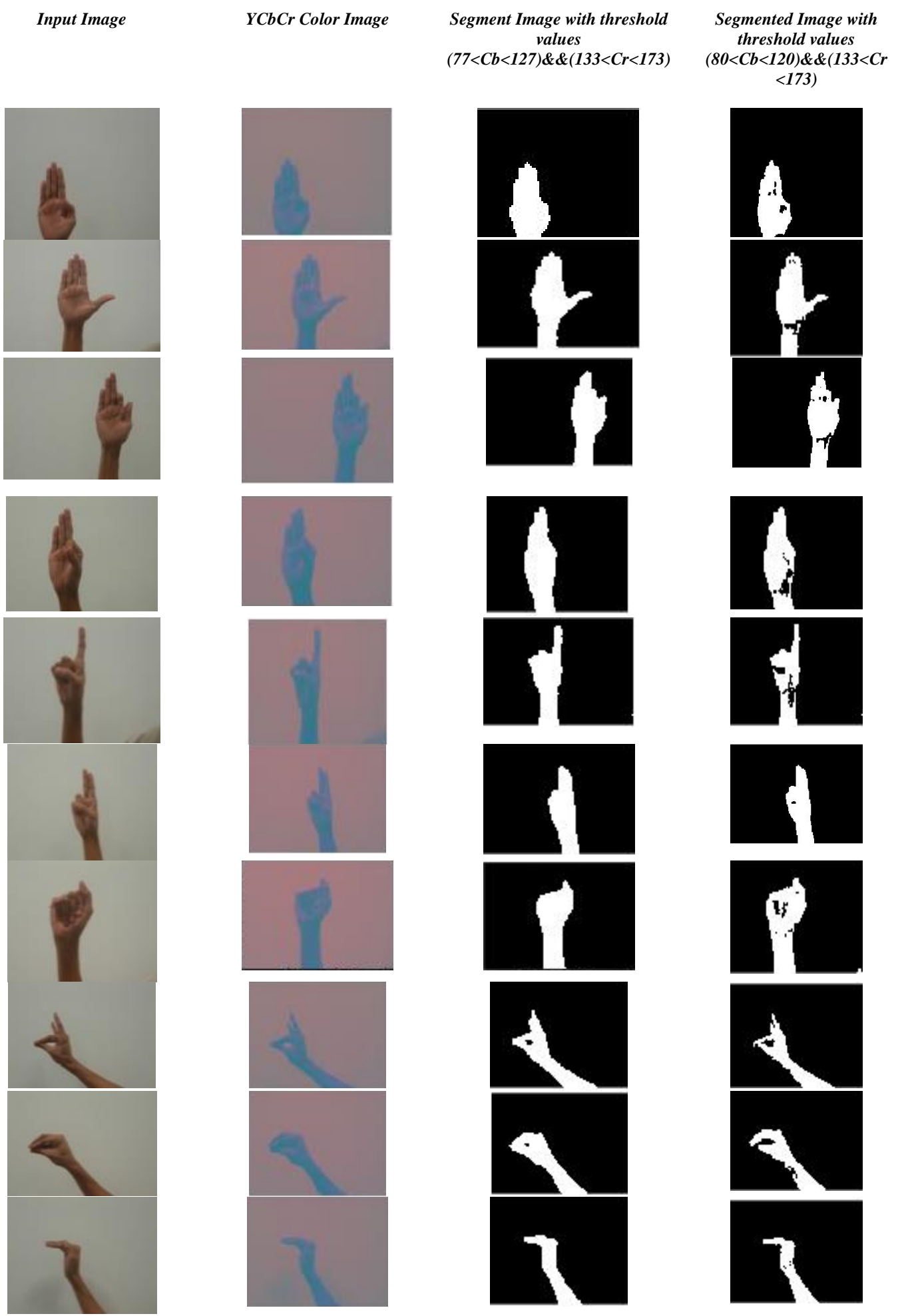

Segmented Image with

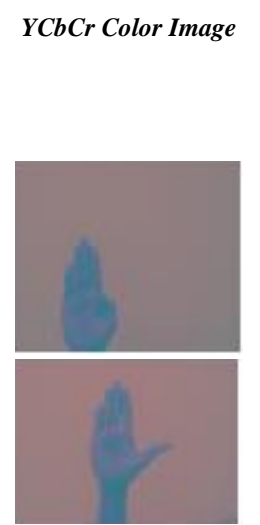
values
$(77<C b<127) \& \&(133<C r<173)$
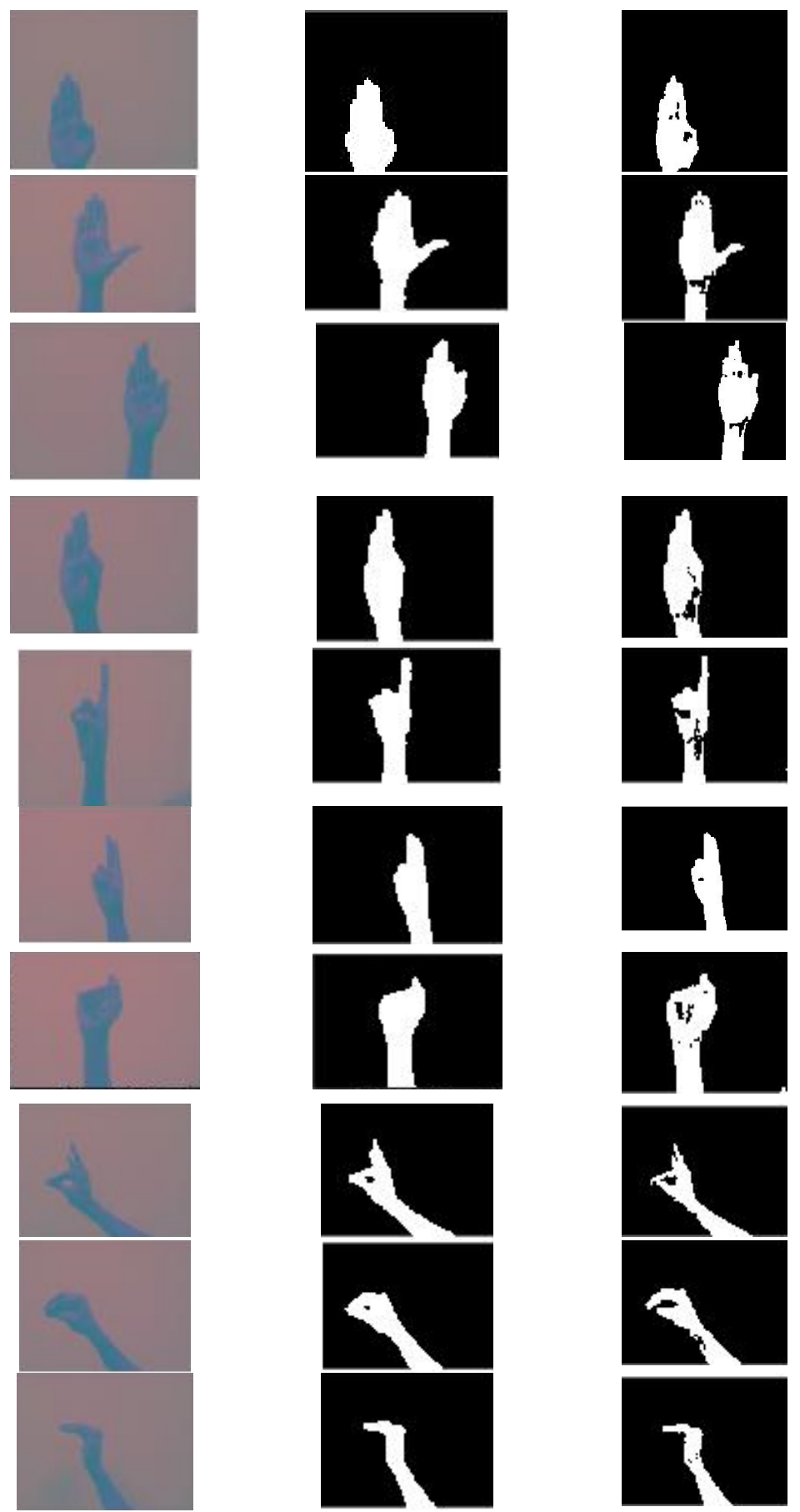

Fig.4. Hand Detection using YCbCr colour model with a uniform background.

\section{threshold values}

$(Y>80) \& \&(85<C b<135)$ $\& \&(135<C r<180)$
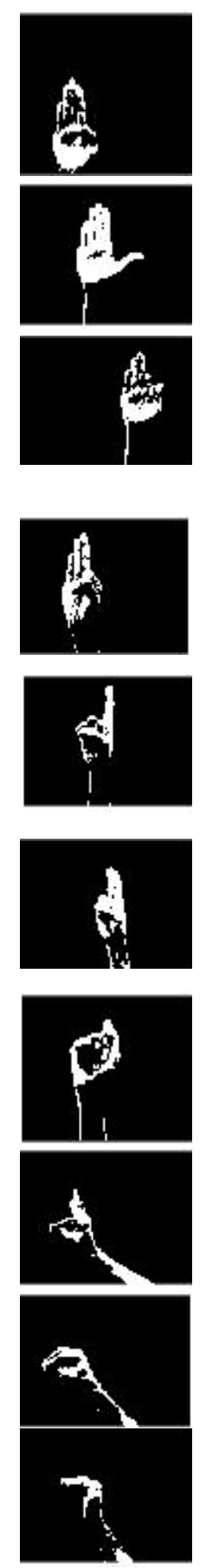
Input Image

YCbCr Color Image
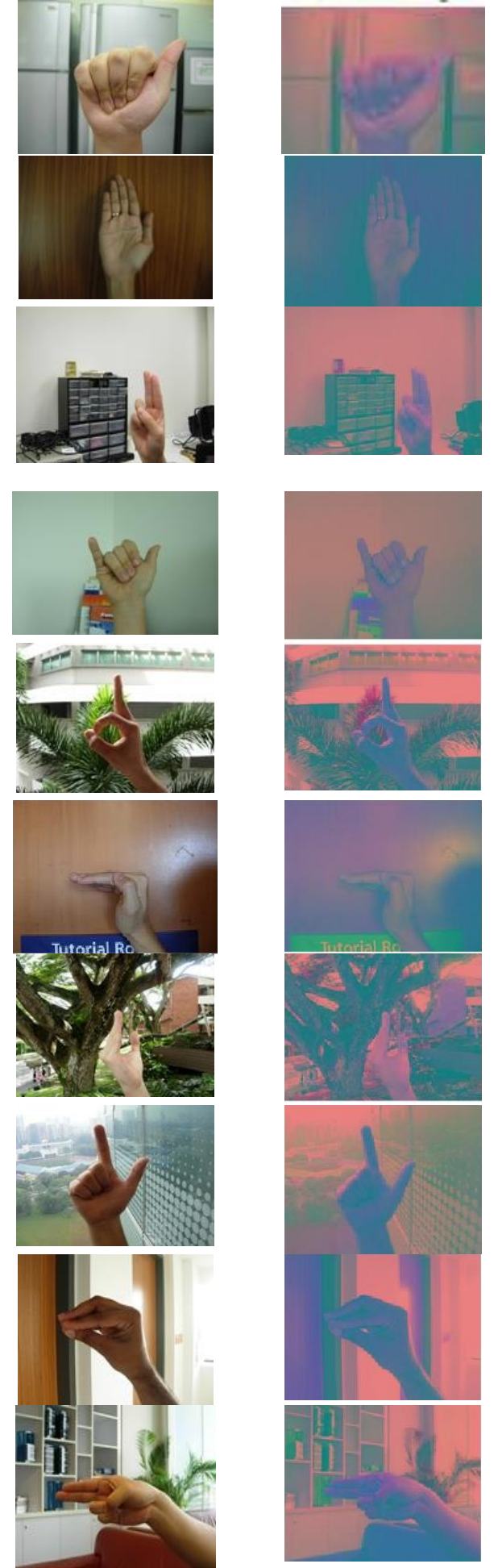

Segment Image with threshold values $(77<C b<127) \& \&(133<C r<1$ 73)
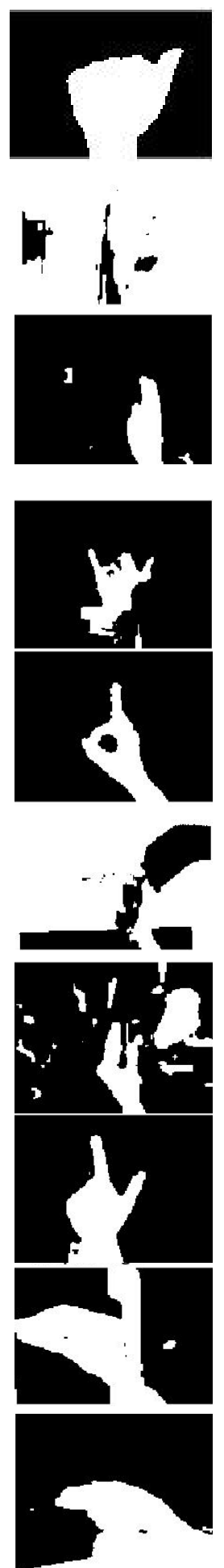
Segmented Image with
threshold values $(80<C b<120) \& \&(133<C r<1$

73)
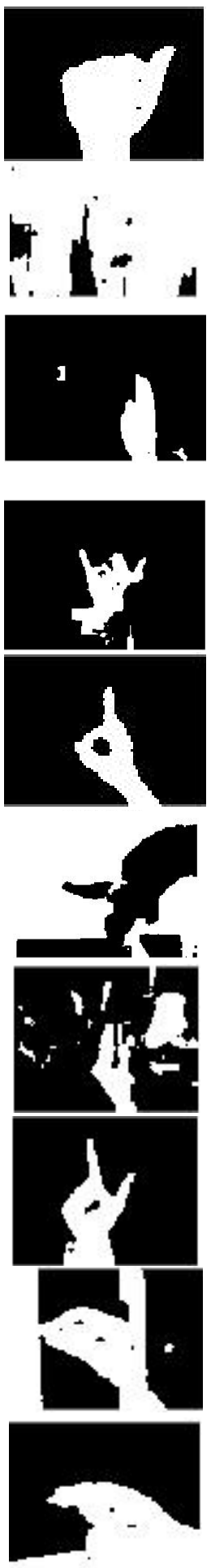

Segmented Image with threshold values $(Y>80) \& \&(85<C b<135)$

$\& \&(135<C r<180)$
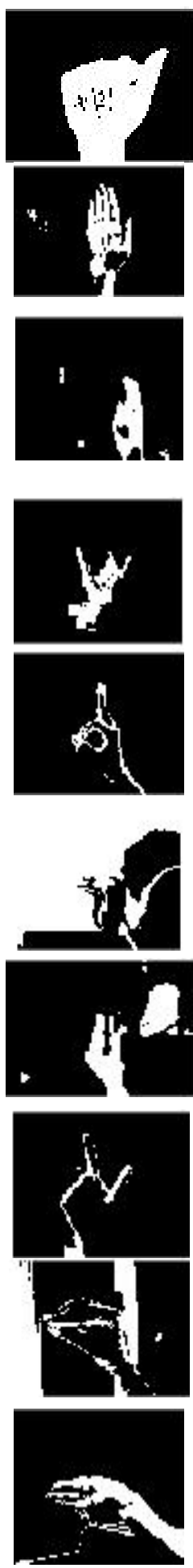

Fig.5. Hand Detection using YCbCr colour mode with complex background 


\section{REFERENCES}

[1] K. B. Shaik, P. Ganesan, V. Kalist, B. S. Sathish, \& J. M. M Jenitha (2015). Comparative study of skin color detection and segmentation in $\mathrm{HSV}$ and $\mathrm{YCbCr}$ color space. Procedia Computer Science, 57, 41-48.

[2] T.J. McBride, N.Vandayar, K.J.Nixon (2019). A Comaprison of Skin Detection Algorithm for Hand Gestrue Recognition.

[3] .D.H Nguyen, T.H. Le, T.H.Tran, H. Vu, T.L.Le, H.G. Doan (2018, October). Hand segmentation under different viewpoints by combination of Mask R-CNN with tracking. In 2018 5th Asian Conference on Defense Technology (ACDT)(pp. 14-20). IEEE.

[4] Q.Zhang, M. Yang, K.Kpalma, Q.Zheng, X.Zhang (2018). Segmentation of Hand Posture against Complex Backgrounds Based on Saliency and Skin Colour Detection. IAENG International Journal of Computer Science, 45(3).

[5] R. F. Rahmat, T. Chairunnisa, D. Gunawan, \& O. S. Sitompul (2016, August). Skin color segmentation using multi-color space threshold. In 2016 3rd International Conference on Computer and Information Sciences (ICCOINS) (pp. 391-396). IEEE.

[6] S. Thakur, S. Paul, A. Mondal, S. Das, \& A. Abraham (2011, December). Face detection using skin tone segmentation. In 2011 World Congress on Information and Communication Technologies (pp. 53-60). IEEE.

[7] S. L. Phung, A. Bouzerdoum, \& D. Chai (2005). Skin segmentation using color pixel classification: analysis and comparison. IEEE Transactions on Pattern Analysis \& Machine Intelligence, (1), 148-154.

[8] S. L. Phung, A. Bouzerdoum, \& D. Chai (2003, July). Skin segmentation using color and edge information. In Seventh International Symposium on Signal Processing and Its Applications, 2003. Proceedings. (Vol. 1, pp. 525528). IEEE.

[9] G. Kukharev, \& A. Nowosielski (2004). Visitor identification-elaborating real time face recognition system.

[10] D. Chai, \& K. N. Ngan (1999). Face segmentation using skin-color map in videophone applications. IEEE Transactions on circuits and systems for video technology, 9(4), 551-564.

[11] Y. Xu, \& G. Pok, (2017). Identification of Hand Region Based on $\mathrm{YCgCr}$ Color Representation. International Journal of Applied Engineering Research, 12(6), 10311034.

[12] W. Wang, \& J. Pan (2012, July). Hand segmentation using skin color and background information. In 2012 International Conference on Machine Learning and Cybernetics (Vol. 4, pp. 1487-1492). IEEE.

[13] S. Patidar, \& D. C. Satsangi (2013). Hand segmentation and tracking technique using color models. International Journal of Software \& Hardware Research in Engineering, 1(2), 18-22.

[14] S. Rungruangbaiyok, R. Duangsoithong, \& K. Chetpattananondh (2015, June). Ensemble Threshold Segmentation for hand detection. In 2015 12th International Conference on Electrical Engineering/ Electronics, Computer, Telecommunications and Information Technology (ECTI-CON)(pp. 1-5). IEEE.
[15] X. Yingxin, L. Jinghua, W. Lichun, \& K. Dehui (2016, December). A robust hand gesture recognition method via convolutional neural network. In 2016 6th International Conference on Digital Home (ICDH) (pp. 64-67). IEEE.

[16] M. R Tabassum, A. U. Gias, M. Kamal, H. M. Muctadir, M. Ibrahim, A. K. Shakir, \& M. Islam (2010). Comparative study of statistical skin detection algorithms for sub-continental human images. arXiv preprint arXiv:1008.4206.

[17] L. Y. Deng, J. C. Hung, H. C. Keh, K. Y. Lin, Y. J., \& N. C. Huang, (2011). Real-time hand gesture recognition by shape context based matching and cost matrix. Journal of networks, 6(5), 697.

[18] U. Ahlvers, R. Rajagopalan, \& U. Zölzer (2005, September). Model-free face detection and head tracking with morphological hole mapping. In 2005 13th European Signal Processing Conference (pp. 1-4). IEEE.

[19] D. L. Lee \& W. S. You (2017). Recognition of complex static hand gestures by using the wristband-based contour features. IET Image Processing, 12(1), 80-87.

[20] J. Sahoo, S. Ari, \& D. Ghosh (2018). Hand Gesture Recognition using DWT and F-ratio Based Feature Descriptor. IET Image Processing.

[21] Z. Qiu-yu, L. Jun-chi, Z. Mo-yi, D. Hong-xiang, \& L. Lu (2015). Hand gesture segmentation method based on $\mathrm{YCbCr}$ color space and $\mathrm{K}$-means clustering. International Journal of Signal Processing, Image Processing and Pattern Recognition, 8(5), 105-116.

\section{Authors' Profiles}

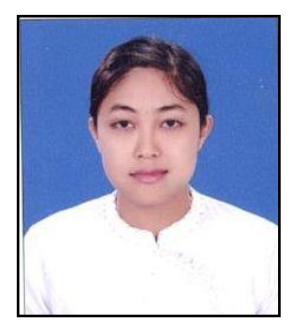

Phyu Myo Thwe, female, Assistant Lecturer, Her research interests include digital image processing and Deep Learning. She got B.C.Sc at 2009 and B.C.Sc (Hons:) at 2010 from the University of Computer Studies, Monywa, Myanmar. She also got M.C.Sc(Credit) degree from the University of Computer Studies, Monywa, Myanmar at 2012. Now, she is currently working toward the Ph.D. degree in the University of Computer Studies, Mandalay, Myanmar.

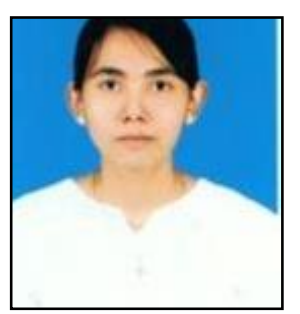

May The 'Yu, female, is an Associate Professor, Faculty of Information Science, University of Computer Studies, Mandalay, Myanmar. She got Ph.D. degree from University of Computer Studies, Yangon, Myanmar at 2014. Her research interest is Image Processing. 
How to cite this paper: Phyu Myo Thwe, May The' Yu, "Analysis on Skin Colour Model Using Adaptive Threshold Values for Hand Segmentation", International Journal of Image, Graphics and Signal Processing(IJIGSP), Vol.11, No.9, pp. 25-33, 2019.DOI: $10.5815 /$ ijigsp.2019.09.03 